\title{
TerraLook: GIS-Ready Time-Series of Satellite Imagery for Monitoring Change
}

TerraLook is a joint project of the U.S. Geological Survey (USGS) and the National Aeronautics and Space Administration (NASA) Jet Propulsion Laboratory (JPL) with a goal of providing satellite images that anyone can use to see changes in the Earth's surface over time. Each TerraLook product is a userspecified collection of satellite images selected from imagery archived at the USGS Earth Resources Observation and Science (EROS) Center. Images are bundled with standards-compliant metadata, a world file, and an outline of each image's ground footprint, enabling their use in geographic information systems (GIS), image processing software, and Web mapping applications. TerraLook images are available through the USGS Global Visualization Viewer (http://glovis.usgs.gov).

Images in a TerraLook collection are rendered in simulated natural color and distributed in JPEG format. The JPEG file format and simulated natural color scheme were selected to make TerraLook images accessible to a wide audience. TerraLook JPEG files are small, which makes them quick to download and display using Web browsers and other commonly available software packages. Simulated natural colors are generally easy to interpret and understand. The simulated natural colors in TerraLook images are consistently processed so that images from five time periods-circa 1975, 1990, 2000, 2005, and the present—can be compared through time.

TerraLook images are drawn from two data sets archived at the USGS EROS. One is the Global Land Survey Landsat data, a set of preselected, nearly cloud-free images for the 1975, 1990, 2000, and 2005 time periods. Data were recorded by three different Landsat sensors: the Multispectral Scanner (MSS) on Landsats 1, 2, and 3; the Thematic Mapper (TM) on Landsats 4 and 5; and the Enhanced Thematic Mapper Plus (ETM+) on Landsat 7. TerraLook's second data source is the collection of over 1.5 million Advanced Spaceborne Thermal Emission and Reflection Radiometer (ASTER) images stored and distributed by the USGS EROS.

TerraLook viewer software has been developed to allow users to view, compare, and annotate TerraLook images. The trilingual (Spanish, French, and English), open source TerraLook viewer is available from Source Forge (http://terralook. sourceforge.net). The viewer allows images to be displayed by selecting an image footprint. The TerraLook viewer has special tools that make it easy to study the Earth through time and construct virtual mosaics. Layers can be created interactively to store annotation and map data, such as park or urban boundaries, or new information, such as the location of sample sites.

TerraLook builds on the success of NASA JPL's Protected Area Archive project (http://asterweb.jpl.nasa.gov/paa.asp) with additional support from The Nature Conservancy, the World Bank Development Grant Facility Program's InterAmerican Biodiversity Information Network Connectivity

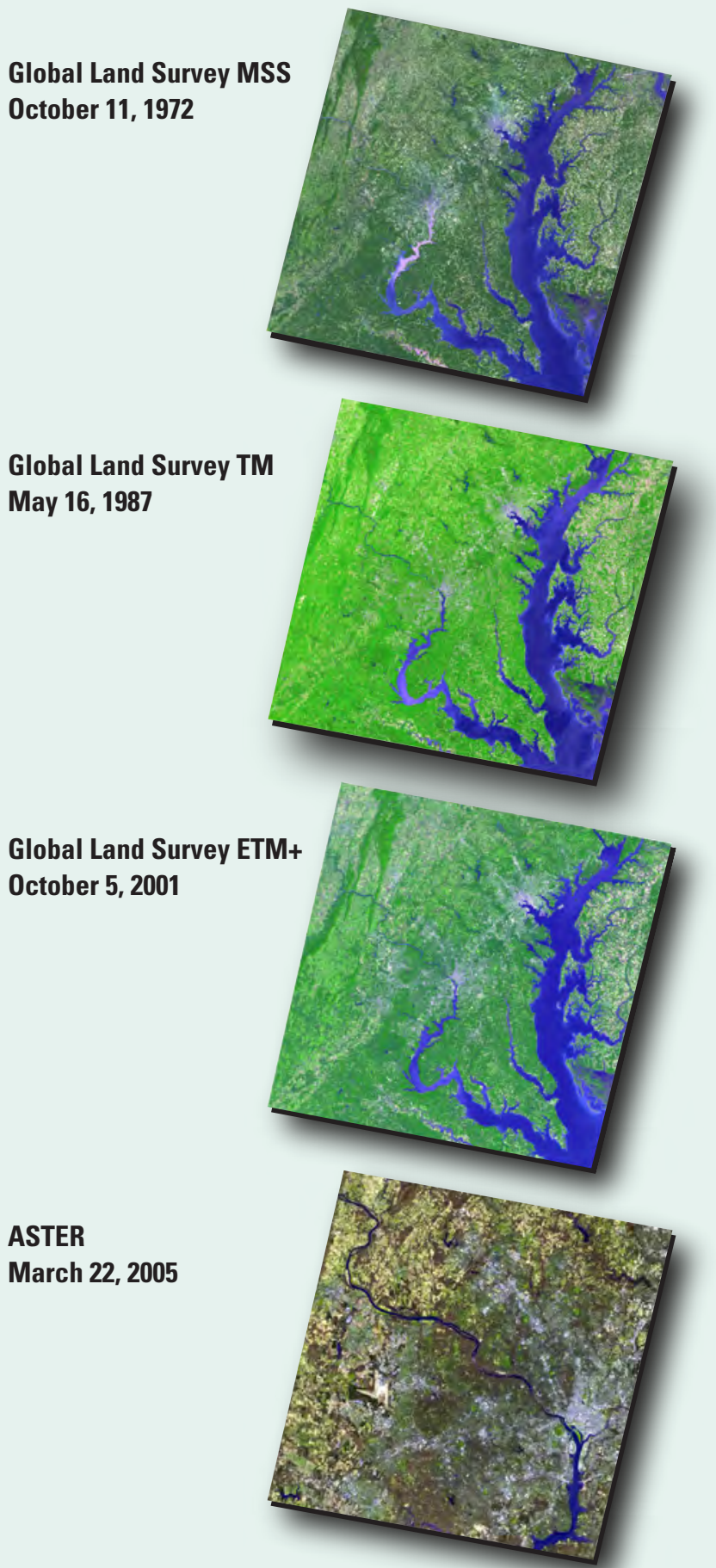

Development near Baltimore, Maryland, and Washington, D.C., intensified between 1972 and 2005. The Dulles International Airport west of Washington, D.C., opened in 1962. In 1972, it was still surrounded by farmland and forest. 
Global Land Survey MSS

February 23, 1973

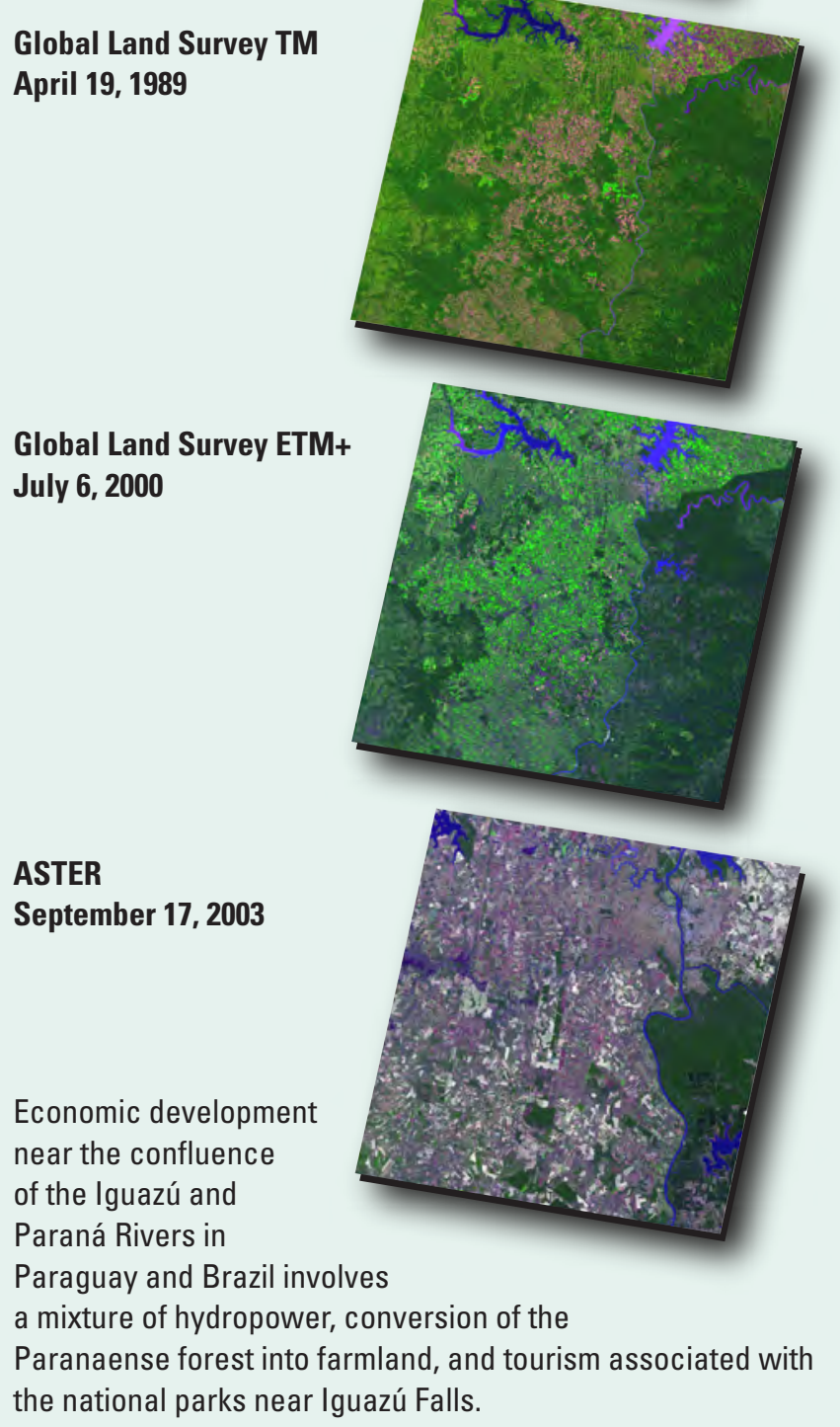

Project, the U.S. State Department, the Gordon and Betty Moore Foundation Amazon Protected Area Network project, and the International Union for the Conservation of Nature and Natural Resources (IUCN; the World Conservation Union). Feedback from the conservation community guided the development of the TerraLook product and viewer, which will continue to evolve as the project reaches out to an even wider user community.

\section{To Search and Order TerraLook Data:}

USGS Global Visualization Viewer (GloVis): http://glovis.usgs.gov/

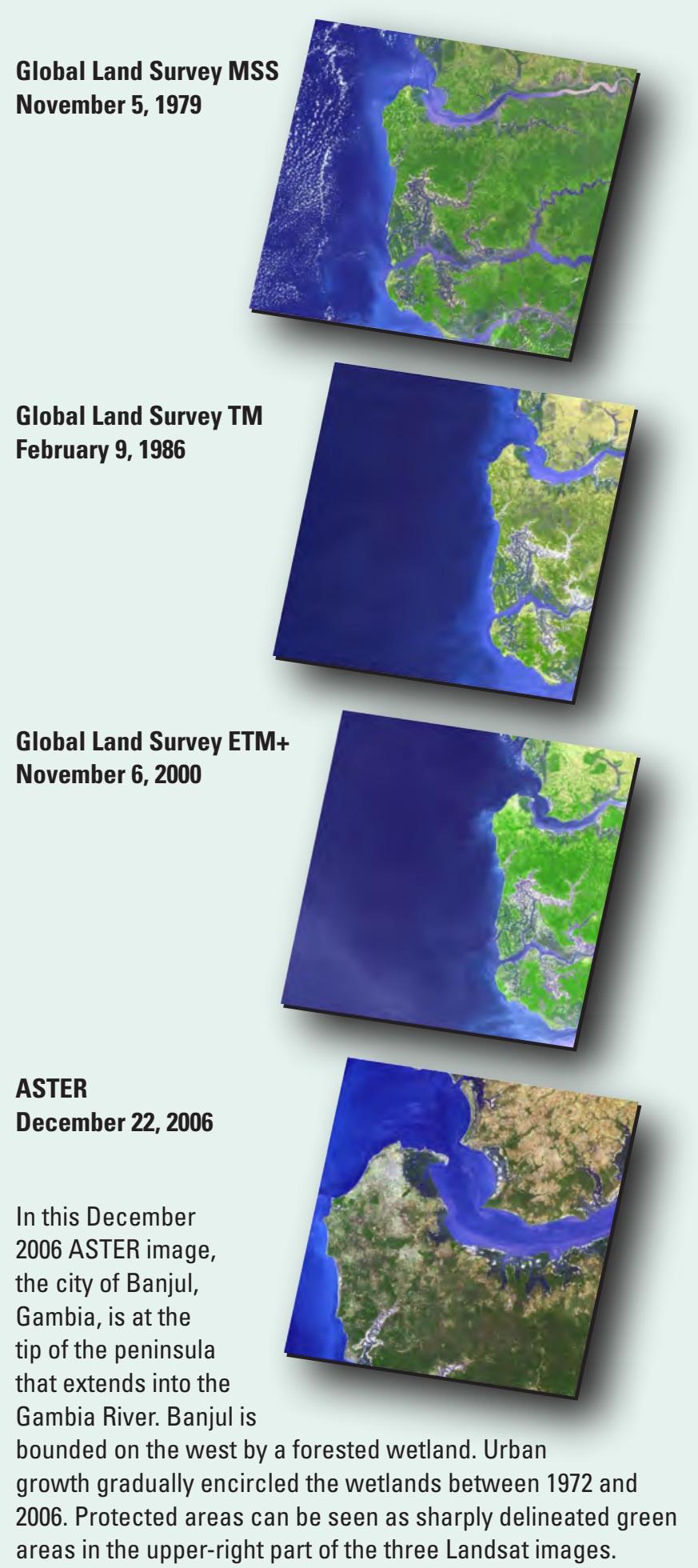

\section{For More Information on TerraLook and the USGS EROS:}

TerraLook Web site: http://terralook.cr.usgs.gov/ USGS EROS Web site: http://eros.usgs.gov/
U.S. Geological Survey

Earth Resources Observation and Science (EROS) Center 47914 252nd Street Sioux Falls, SD 57198
Phone: (800) 252-4547

Phone: (605) 594-6151

TDD: (605) 594-6933

Fax: (605) 594-6589

E-mail: custserv@usgs.gov
Any use of trade, product, or firm names is for descriptive purposes only and does not imply endorsement by the U.S. Government. 